\title{
La enfermería como apertura a la interdisciplina
}

Nursing as an opening to interdisciplinarity

A enfermagem como abertura à interdisciplinar

Fecha de recepción: 27 de junio de 2017

Fecha de evaluación: 15 de septiembre de 2017

Fecha de aceptación: 31 de octubre de 2017

Disponible en línea: 21 de noviembre de 2017

José Enrique Gómez Álvarez*

DOI: https://doi.org/10.18359/rlbi.3162

\author{
Cómo citar: \\ Gómez Álvarez, J. E. (2018). La enfermería como \\ apertura a la interdisciplina. Revista Latinoamerica- \\ na de Bioética, 18(1), 191-204. \\ Doi: https://doi.org/10.18359/rlbi.3162
}
* Doctor en Filosofía, Universidad de Navarra, maestro en Gerontología Social y enfermero auxiliar. Profesor investigador en la división de Bioética del Centro de Investigación Social Avanzada (Cisav), Ciudad de México. Correo electrónico: jegomezalvarez@yahoo.com. ORCID: https://orcid.org/0000-0002-8964-2207




\section{Resumen}

Este artículo reexamina los conceptos interdisciplinario, complejo, el cuidado y enfermería. Lo que busca es argumentar que la noción de enfermería y el cuidado, como propiedad esencial de esta, siempre lleva a ser una interdisciplina. Se parte de la noción de holístico, que suele usarse como concepto contrario de específico en las intervenciones de enfermería, y que se relaciona con el objeto formal propio de esta: el cuidado. A partir de estos dos elementos se propone y defiende que el mejor término para describir su objeto es complejo, considerando la propuesta de Rolando García de sistema complejo, para defender a la enfermería como un todo complejo, incluso al compararla con los elementos teóricos (como los de la enfermería) y las evidencias empíricas de testimonios de la práctica de la enfermería. Por último, se muestra que la ética es un ejemplo de esa realidad compleja de la profesión del cuidar. Se concluye con una reflexión final.

Palabras clave: lógica modal, enfermería, cuidado, epistemología del cuidado, ontología del cuidado.

\section{Abstract}

This paper reexamines the concepts of interdisciplinary, complexity, care and nursing. The main target is to argue that the notion of nursing and care, as an essential property of this, always leads to the interdisciplinary approach. It is based on the holistic idea of care which is used as an opposite concept of specific nursing interventions, and which is related to the formal object of this: care. From these two elements, it is proposed and defended that the best term to describe its purpose is complex, considering Rolando Garcia's proposal of a complex system. To support nursing a complex whole, even when it's compared with theoretical elements (such as nursing elements) and the empirical evidence of the nursing practice. Finally, it is shown that ethics is an example of that complex reality of the caring profession. It concludes with a final reflection.

Keywords: Modal logic, nursing, care, epistemology of care, ontology of care.

\section{Resumo}

Este artigo reexamina os conceitos, interdisciplinar, complexo, o cuidado e enfermagem. O que ele procura é argumentar que a noção de enfermagem e o cuidado, como uma propriedade essencial dela, sempre leva a ser uma interdisciplina. A partir da noção de holístico, que é frequentemente usada como um conceito oposto de específico nas intervenções de enfermagem, e que está relacionado com o objeto formal próprio desta: o cuidado. A partir desses dois elementos, é proposto e defende que o melhor termo para descrever seu objeto é complexo, considerando a proposta de Rolando García de sistema complexo, para defender à enfermagem como um todo complexo, mesmo quando comparado com os elementos teóricos (como os da enfermagem) e as evidências empíricas de testemunhos da prática de enfermagem. Finalmente, é demonstrado que a ética é um exemplo dessa complexa realidade da profissão de cuidar. Conclui-se com uma reflexão final.

Palavras-chave: lógica modal, enfermagem, cuidado, epistemologia do cuidado, ontologia do cuidado. 


\section{Abierto, holístico y cuidado}

Desde hace tiempo se han realizado algunas reflexiones en torno a la enfermería y la ética (Gómez, 2014), y a las prácticas profesionales del autor de este trabajo en primero y segundo nivel de atención. Las posteriores actividades en clínica han mostrado algo bien sabido, pero no del todo clarificado: la práctica de la enfermería es un todo holístico. Estas reflexiones han surgido, por una parte, de una investigación realizada en torno a los programas de estudio de bioética y su visión metodológica de cada una de ellas. Esa investigación inició en 2011 y se concluyó en 2017. Este artículo conecta parte de esa reflexión junto con el proceso personal del autor en torno a lo que implica la actividad profesional de la enfermería.

De regreso al tema de lo holístico, más allá de la frase correcta, aunque ambigua, ¿qué implica? Si revisamos el término en PubMed, por ejemplo, se encuentran que se rebasan los 3000 documentos que llevan esa expresión en el título (PubMed, 2017), y si lo restringimos al concepto de nursing, se reduce a poco menos de 400 títulos. ¿Qué se descubre al analizarlos?

Se puede empezar con el concepto de cuidado inclusivo. Existen autores de corte religioso que, preocupados por la salud, pero al mismo tiempo viendo al hombre en todas sus dimensiones, apuntan el papel de la vida religiosa sin perder el rigor científico. Así, Jawad y Kalra, en Ramadan and diabetes: Holistic trial design (2016), apuntan lo siguiente:

\footnotetext{
Along with bio medical markers such as blood pressure, weight, blood glucose and lipids, holistic studies on diabetes management during Ramadan should take spiritual health into account. Validated instruments should be researched as possible tools to
}

aid in pre-Ramadan counseling, risk stratification and decision making. They should also as means of quantifying and monitoring spiritual bliss before, during and after Ramadan. The results thus obtained should be correlated with biomedical markers, to assess if spiritual wellbeing, biomedical (physical) health, and laboratory values go hand in hand. Such holistic research will reinforce the complementary nature of science and religion, and help clinicians in their pursuit to achieve holistic health for persons living with diabetes who wish to observe Ramadan in their guidance. (p.792) (el subrayado es mío).

En este documento, holístico hace alusión a no dejar de lado todas las necesidades humanas, y debe notarse que indican el deber de tomarse como parte del cuidado de salud.

Un modo de entenderlo por analogía es determinar el uso de las expresiones del cuidado materno o paterno: se es cocinero, doctor o consejero, ya que se realiza "de todo" al ejercer la paternidad-maternidad (Fursterberg, 2017). ${ }^{1} Y$, en este aspecto, la enfermería se le parece, porque, para decirlo de un modo sencillo, es holística.

Por otra parte, es necesario tener cautela con el uso de ese término, ya que parece una categoría ambigua que suele apuntar a "todo y nada”. ¿No es convertir la práctica científica y disciplinaria en una mezcla de saberes o palabrería? Podría caerse, entonces, en lo que Sokal y Bricmont denuncian en Impos-

1 No se hace esto en defensa del "paternalismo" entendido como el "estar encima" del paciente decidiendo por él. El sentido es que el cuidado de la salud es multifactorial y requiere habilidades no sólo técnicas, sino comunicacionales, organizacionales y disposición de entrega personal. 
turas intelectuales (1999); es decir, convertir lo "interdisciplinario" del cuidado (papel central de la enfermería) en "una erudición superficial lanzando, sin el menor sonrojo, una avalancha de términos técnicos en un contexto en el que resultan absolutamente incongruentes" (p. 4).

Los riesgos en el abuso o mal uso del lenguaje siempre han existido; por ejemplo, Paul Feyerabend (1993) tiene en su haber un ensayo exquisito donde denuncia, de modo semejante a Sokal y Bricmont, la presentación lingüística de problemas no científicos con una terminología que solo oscurece el sentido ordinario de los términos.

Por tanto, en este artículo no se excluyen esas advertencias, sino más bien se busca afrontarlas con el fin de que sirvan de apoyo para entender mejor cuál es el papel de lo interdisciplinario en la enfermería y su apertura a otras disciplinas que convierten el cuidar en algo más que una profesión. Es crucial referirme a nociones explicadas, pero de acuerdo con la visión de una razón ampliada, ya que considero que la verdad no debe confundirse con erudición.

Una forma de buscar soluciones más completas a los problemas humanos en las profesiones sanitarias es desde ellas mismas $\mathrm{y}$ vistas como un todo. Asimismo, en lugar de separar los problemas en clínicos, trabajo social y derechos humanos, etcétera, se busca resolverlo en conjunto. Esto lleva a plantear muchas interrogantes. ¿No es eso como una mezcolanza inútil donde las metodologías se borran? (Calderón, 2003), ¿no es eso mismo una especie de desiderátum mal logrado de "convivencia armoniosa de las disciplinas"?

Quizá un modo de entender lo que se plantea es utilizando el término complejidad, el cual, aunque se ha vuelto común en áreas como las organizaciones sociales (teoría de sistemas) o ciertos comportamientos en el campo de la vida animal y humana, ¿sería la respuesta de cómo dialogar las disciplinas sin que aparezca la tentación del dominio de una sobre otra?

Este ensayo defiende que la interdisciplinariedad aún es un camino en construcción, pero crucial para el avance de las ciencias del cuidado, donde se incluyen todas las llamadas "disciplinas sanitarias". Aunque tradicionalmente estas se dividen en compartimentos cerrados: medicina, enfermería, trabajo social, derecho, etcétera, se sugiere replantear los problemas bioéticos, si quiere llamárselos así, de un modo global. Es cierto que algunos autores, como Ana Martha González (2001), han señalado que en el caso de la bioética en realidad se trata de una ética, y que su supuesta originalidad es menos importante de lo que se considera:

Sin duda, desde un punto de vista práctico, los partidarios de constituir a la bioética en una ciencia autónoma pueden alegar a su favor el hecho innegable de que [...] ha generado, en pocas décadas, una reflexión ingente y muy específica, que además de requerir la aplicación de los principios éticos a una materia muy concreta, exige internamente la adopción de una perspectiva multidisciplinar a la hora de afrontar sus problemas específicos.

Aunque esta postura es defendible desde un punto de vista práctico, considero que por sí sola no autoriza a conceder a la bioética un estatuto epistemológico diverso del de la ética. Pues, de una parte, la concreción de su materia no hace de ella una ciencia di- 
versa, ya que también aquí se trata de acciones humanas (por mucho que la materia de estas acciones se circunscriba a un ámbito determinado) y, de otra, su mismo carácter interdisciplinar ?que es hasta cierto punto lo más novedoso de la bioética? no constituye tampoco un motivo suficiente para constituirla en una ciencia independiente. Después de todo, la misma interdisciplinariedad podría verse como una ampliación sistemática de la deliberación que ha de preceder a toda decisión éticamente aceptable, cuya aceptabilidad, en todo caso, corresponde examinar a la ética. (p. 305)

Dicho de otro modo, sin negar que la especificidad de la ética es su acercamiento a la realidad, parece que hoy en día repensar la interdisciplina puede ayudar a mejorar las prácticas y los protocolos de atención.

La Bioética nos ha enseñado, a pesar de la observación indicada por González (2001), que la "consulta" interdisciplinaria es, en muchos asuntos, la única manera de afrontar con rigor epistémico y ético ciertas realidades. Una de ellas es el arte y la ciencia de curar.

\section{El concepto de enfermería}

"El cuidado del hombre" y "el cuidado del ser humano en sus necesidades" son modos donde a veces se incluye la profesión de la enfermería. Si se apoya el concepto en definiciones más formales, podría citarse que:

Los cuidados profesionales enfermeros son todas aquellas formas humanísticas y científicas, aprendidas en forma cognitiva, de ayudar o capacitar a individuos, familias o comunidades para recibir servicios personalizados a través de modalidades cul- turalmente determinadas, técnicas y procesos orientados al mantenimiento $\mathrm{y}$ desarrollo de condiciones favorables de vida y muerte. (Reyes, 2009, p. 73)

Se repite "hasta el cansancio" que el objeto de la enfermería es cuidar, ${ }^{2}$ y se está de acuerdo, pero en el caso de la anterior definición, ¿no se apega a toda disciplina posible?, o en términos lógicos, ¿no es demasiado amplia? ¿Acaso cuando se realiza una actividad, por ejemplo, de abogacía, no se busca "cuidar al otro"?, y en general podríamos ejemplificarlo para muchas áreas.

Un modo de resolver este cuestionamiento es revisar las teorías de la enfermería; de esta manera, al observar la teoría de las necesidades fundamentales, ${ }^{3}$ de Henderson, se descubre que dichas necesidades son humanas o se han humanizado, ya que las primera nueve son de carácter fisiológico, mientras las otras aluden claramente a componentes o necesidades humanas, como la comunicación y la espiritualidad (Younas y Sommer, 2015, p. 444).

Es conveniente resaltar que algunos autores, como Delgado, Hernández y Ostiguín (2007), afirman que:

2 Para las teorías de enfermería, un resumen donde se hace manifiesto el núcleo de la enfermería se encuentra en: http://nursingtheories.weebly. com/florence-nightingale.html

3 Necesidad 1: respirar normalmente; necesidad 2: comer y beber de forma adecuada; necesidad 3: eliminar los desechos corporales; necesidad 4: moverse y mantener una postura adecuada; necesidad 5: dormir y descansar; necesidad 6: elegir la ropa adecuada; necesidad 7: mantener la temperatura corporal; necesidad 8: mantener la higiene corporal; necesidad 9: evitar los peligros del entorno; necesidad 10: comunicarse con los otros; necesidad 11: actuar con arreglo a la propia fe; necesidad 12: trabajar para sentirse realizado; necesidad 13: participar en diversas formas de entretenimiento; necesidad 14: aprender, descubrir o satisfacer la curiosidad. 
La propuesta de Virginia Henderson se considera filosofía debido a que no permite organizar los conceptos y las definiciones para lograr una relación entre sí con el fin de proporcionar una estructura de naturaleza sistemática. La falta de organización de conceptos no permite tener un fundamento para las acciones de Enfermería, que explique en forma sistemática los métodos de atención y prediga los resultados. (p. 25)

Sin embargo, toda teoría conlleva una filosofía o suposición metafísica que le es propia. Lo que interesa resaltar es que la complejidad del cuidado implica siempre "tener a flote" la filosofía, como la denominan estos autores, y los aspectos técnico-científicos para lograr ese propósito del cuidado. No obstante, tienen razón en cuanto a los principios, para llevarlos a la práctica y su integración quedan incompletos como modelo de enfermería, porque pareciera que falta ese medio de acción (el cual, por cierto, se busca en las clasificaciones de NOC, NIC). De ahí que otros autores han propuesto cómo integrar esos principios en la práctica clínica (véase Younas, y Sommer, 2015).

Elíjase una necesidad fisiológica de las indicadas por Henderson: el evacuar o eliminar los desechos. Al analizar los criterios de valoración comunes se puede subdividir en múltiples elementos, como revisar ruidos intestinales o si hay fisuras anales, entre otras, ${ }^{4}$ pero es claro que se debe cuidar más que eso: se cuida a la persona y, en sentido impropio, a la fisura. Así que la propuesta de este autor, no importando si es un modelo de cuidados y aceptando que es una filosofía, se puede tomar con ese sentido etimo-

4 Véase: http://www.ome.es/04_01_desa. cfm?id=424\#ancla3 lógico: sabiduría. Las personas que cuidan de esta manera, al atender una necesidad fundamental, buscan la respuesta en el todo. Si un paciente sufre dolor, se atiende, pero también todo el entorno de sufrimiento, que no es reductible a algunas de las intervenciones de NANDA, por mencionar alguna (Care Plans, 2017).

Otro ejemplo es el siguiente: si se revisan los patrones funcionales de Gordon: percepción de mantenimiento de la salud, patrón de eliminación, actividad, ejercicio, reposo sueño, rol, relaciones, sexualidad, reproducción, adaptación y tolerancia al estrés, y valores y creencias, se observa que cubren una amplia gama de actividad humana, no solo de fisiología. En relación con esto último, los patrones planteados por Álvarez Suárez, Del castillo Arévalo, Fernández Fodalgo y Muñoz Meléndez (2010) advierten:

De la valoración de los patrones funcionales se obtiene una importante cantidad de datos relevantes de la persona (físicos, psíquicos, sociales, del entorno), de una manera ordenada, lo que facilita a su vez el análisis de los mismos [...] Los 11 patrones son una división artificial y estructurada del funcionamiento humano integrado, no deben ser entendidos de forma aislada; la interrelación que se da entre ellos es lógica, las personas somos un TODO sin compartimentar, todo influye en todo (el entorno, la cultura, el nivel social, los valores, las creencias [...]). Algunos patrones comparten información y ciertos datos pueden estar presentes en más de un patrón, no siendo necesario registrarlos de forma repetida. (p. 1)

Lo descrito por lo autores no es complicado, es complejo. La idea del "cuidado" es un todo analógico. Existe un analogado principal y este se 
aplica proporcionalmente: "si la razón análoga se encuentra realizada intrínsecamente en todos los analogados, lo llamamos analogía según la causalidad formal intrínseca. Si, empero, se encuentra intrínsecamente en uno solo y en los demás denominativamente, lo llamamos analogía según la causalidad extrínseca" (González, 1987, p. 182).

"Cuidar" es, por tanto, un elemento análogo a la salud, porque si se retoma a Aristóteles (1987) con el mismo ejemplo, la "salud", se diría que es una analogía de atribución. En sentido estricto, la salud está en cada viviente concreto y la enfermería, al ser una profesionalización del cuidado, es causa en diversas modalidades. ${ }^{5}$ Así, en una técnica como la oxigenoterapia se restablece el equilibrio orgánico, pero en otras, se plantea una causa ejemplar: el modelo de preservar la salud. Así, en los trabajos de educación para la salud, al enseñar a cepillarse correctamente los dientes o cómo realizar un correcto aseo de manos, también se vela por un modelo.

No obstante, este análisis con ejemplos sencillos nos oculta que, al intervenir en algún aspecto del cuidado sanitario, se suele alterar otro para bien o mal del paciente; un ejemplo clásico es la intervención en el área de la salud. En gerontología, al planear un menú adecuado hiperproteico, se encuentra en ocasiones con que el paciente tiene problemas de masticación; entonces lo común es realizar preparados suaves, como purés, pero resulta que al hacer esto la comida pierde textura y sabor, por lo que el anciano, de por sí, con poco apetito, deja de comer. Otro ejemplo sobre este mismo tema es que el alimento tiene buen sabor, aunque la apariencia resulta repulsiva para el anciano. Todo

5 Usando la terminología aristotélica: formal, ejemplar, final y eficiente. ello deviene en considerar que la intervención del cuidado en enfermería es compleja (Bernal-Orozco, Bizmanos y Celis de la Rosa, 2008), porque la habilidad de mantener o considerar "el todo es mayor que la suma de sus partes", es la postura auténtica de lo holístico. En este sentido, dicha postura es ontológica y posee supuestos fuertes en relación con el hombre. Así, Zamanzadeh, Valizadeh y Keogh (2015) señalan:

The relationship between the providers of health care and the patients is based on respect, relative openness, equality, and mutuality and patients participate in decision making in this kind of caring. Providers of holistic care consider a patient as a whole within his/her environment and realize that a patient is made up of a body, mind, and spirit... Holistic care increases self-awareness and self confidence in patients and causes nurses to better understand the effects of an illness on a person's entire life and his/her true needs. It also improves harmony between mind, body, emotions, and spirit in an ever-changing environment. (pp. 214 y 215) (el subrayado es del autor)

Se han subrayado elementos que resultan casi obvios, pero ante una insistencia contemporánea en el respeto a la autonomía del paciente, que puede llegar a convertirse en un voluntarismo subjetivista, se piensa que los autores, con atino, defienden un mínimo de objetividad en el cuidado de los pacientes. Se debe utilizar un elemento antropológico como criterio, aunque no puramente subjetivo: si la persona tiene los elementos constitutivos, no solo materiales, sino realmente un espíritu (sin metáfora), entonces el carácter o la orientación del cuidado lleva a plantear un modo parcialmen- 
te diferente de atención. Si el bienestar humano es más que controlar los aspectos subjetivos del cuidado, ya que el "todo es mayor que la suma de las partes", es necesario considerar protocolos personalizados de verdad respecto a las personas. Así, en el caso del control del dolor habrá casos en los que no deba sedarse a un paciente debido a su necesidad de mantenerse despierto para cumplir sus obligaciones religiosas, aun a costa del acortamiento de su vida. De igual forma, si un paciente se encuentra en cuidados intensivos, es quizá conveniente, respecto a las cuestiones espirituales, como en el catolicismo, la unción de los enfermos, que uno no se apegue a los horarios restringidos de visitas.

De nuevo surge la importancia de la bioética y su conexión con la enfermería. No son un complemento una de otra, sino se superponen de un modo en que, aunque conceptualmente las podemos y debemos distinguir, en la realidad de razón práctica conviven y no pueden estar una sin la otra. Así en el ejemplo citado, podemos distinguir y hablar todos los involucrados con un paciente de la autonomía (distinción conceptual), pero la decisión de cuidarlo implica que al pensar como enfermero se piensa como bioeticista, porque el cuidado humano es polifuncional.

En suma, supone reconocer que existe un verdadero bien para la persona, aun cuando se me pida lo contrario, como la práctica de la eutanasia. Así entendido, el "cuidar" es una noción metafísica, ontológica; es una atención holística que busca el bien propio del hombre y, en consecuencia, es multidimensional, pero no caprichosa. Aunque por necesidades hospitalarias la enfermería se ha subespecializado, por lo que es común, por ejemplo, que existan equipos dedicados solo a los accesos venosos, de modo que se aumen- te la eficiencia y se disminuyan riesgos de infecciones asociadas al cuidado de la salud, no desaparece el carácter holístico de este. Entonces, de nuevo surge la duda: ¿qué peculiaridad tendría la enfermería? Esta pregunta puede responderse después de revisar el concepto complejo.

\section{Lo complejo}

Un error común, quizá, es afirmar que lo complejo es complicado. Es cierto que a menudo el acceso a lo real es complicado, porque tiene muchos elementos que podemos separar y distinguir; sin embargo, de ahí no deriva la complejidad. Esta hace alusión a fenómenos (sería mejor expresar realidades) que son irreductibles en su explicación y abordaje en una sola disciplina:

Many phenomena have stymied the reductionist program: the seemingly irreducible unpredictability of weather and climate; the intricacies and adaptive nature of living organisms and the diseases that threaten them; the economic, political, and cultural behavior of societies; the growth and effects of modern technology and communications networks; and the nature of intelligence and the prospect for creating it in computers. The antireductionist catch-phrase, "the whole is more than the sum of its parts," takes on increasing significance as new sciences such as chaos, systems biology, evolutionary economics, and network theory move beyond reductionism to explain how complex behavior can arise from large collections of simpler components. By the mid-twentieth century, many scientists realized that such phenomena cannot be pigeon holed into any single discipline but require an 
interdisciplinary understanding based on scientific foundations that have not yet been invented. Several attempts at building those foundations include (among others) the fields of cybernetics, synergetics, systems science, and, more recently, the science of complex systems. (Mitchel, 2009, p. 3)

Rolando García (2009) expresa esto de un modo iluminador para los propósitos de este ensayo:

\begin{abstract}
En nuestra concepción de los sistemas complejos, lo que está en juego es la relación entre el objeto de estudio y las disciplinas a partir de las cuales realizamos el estudio. En dicha relación, la complejidad está asociada con la imposibilidad de considerar aspectos particulares de un fenómeno, proceso o situación a partir de una disciplina específica. (p. 21) (el subrayado es del autor)
\end{abstract}

En otros términos, en el "mundo real" las situaciones y los procesos no se presentan de manera que puedan clasificarse por su correspondencia con alguna disciplina en particular. En ese sentido, se hablaría de una realidad compleja, ya que un sistema complejo es una representación de un recorte de esa realidad, conceptualizado como una totalidad organizada (de ahí la denominación de sistema), en la cual los elementos no son "separables" y, por tanto, no pueden estudiarse aisladamente.

Ya se han subrayado los dos elementos que ayudarán a mostrar que el "curar" es, desde ese punto de vista y con el componente metafísico ya comentado de la analogía, una realidad compleja.

Por una parte, se señala el carácter indivisible de lo complejo o su expresión en el lenguaje en términos negativos. Digamos que se podría expresar en la proposición modal (Redmond, 1999), relacionándola con el curar así:

(p1): no es posible considerar aspectos particulares del curar a partir de una disciplina específica.

Lo que equivale a:

(p2): no necesariamente se consideran aspectos particulares del curar a partir de una disciplina específica.

Por lo tanto, si todo sistema complejo es conceptualizar una realidad como totalidad organizada, entonces (nótese que este sentido de complejidad está alejado de lo ya señalado en la introducción: ese abuso lingüístico donde con el solo hecho de mencionar algo o usar una metáfora) se cree estar haciendo algo complejo. De esta manera, la respuesta al estatus de la enfermería es que no es una disciplina, siempre ha sido una actividad y su propia teorización fue el intento de sistematizarla y presentarla como un sistema irreductible a una ciencia particular. La enfermería no es una medicina de segunda, ni solo una persona que ayuda de buena voluntad; tampoco es un conjunto de técnicas y procedimientos por sí sola. Cuando se le presenta así crea el dilema o la paradoja de que se disuelve en las manos; aunque es cierto que la enfermería ya no se puede identificar con algo, sin embargo, queda claro que sigue siendo algo real.

Con este marco conceptual se entienden perfectamente los diversos artículos e investigaciones que plantean la necesidad de incluir todo en el trabajo de enfermería. Por ejemplo, aparte de los autores musulmanes mencionados, Washington, Oliver, Cage, Albright, (2017), en A Multimethod Analysis of Shared Decision-Making in Hospice Interdisciplinary Team Meetings Including Fami- 
ly Caregivers, sorprende cuando señalan que al incluir a los familiares en un modelo de decisión de enfermería:6 "family members' inclusion on health care teams impacts the team's context, structure, and processes which, in turn, affect team outcomes" (p. 3). Este es un magnífico ejemplo de complejidad y equilibrio sistémico, ya que el todo es afectado; como el todo de un modo no linear. Dicho en otros términos, los criterios señalados por Rolando García (2009) nos sirven para interpretar cuando estamos ante una realidad y una interdisciplina.

En la literatura revisada podemos observar más ejemplos de la necesidad de implementar lo holístico (ver tabla 1).

Esto es un ejemplo de lo que significa perplejidad moral, que no es un mero estado de duda. La perplejidad oscila entre decidirse por lo uno y lo otro, pero nadie puede instalarse, éticamente hablando, en la contradicción, ya que se tiene que resolver de algún modo. En estos casos, los principios de perplejidad moral son criterios de decisión, porque su finalidad es disminuir el mal o daño y fomentar el bien. A menudo se recurre a ellos para enfrentarse a situaciones límite y tomar una decisión ética donde los actos maleficentes se hacen presentes. Las decisiones éticas son, pues, complejas en el área de la bioética: no pueden resolverse con el acercamiento de una sola disciplina, requieren el concurso de diversas voluntades que ven el todo, sin renunciar a lo suyo o la parte, reconociendo que el bien del que está enfrente, el paciente no solo él o ella, no es la suma de acciones del trabajador social, el médico, el seguro social o la familia, sino que, como todo digno, sobrepasa a las meras decisiones.

6 Shared Decision Making (SDM) o Decision compartida (en el fondo un curar compartido).
Las acciones que condicionan el florecimiento humano son un elemento crucial que tiene un significado en la dignidad humana, no en cuanto al ser viviente, porque si así fuera el objetivo de las ciencias de la salud sería mantener la metabolización biológica a toda costa, y de lo que se trata es de mantener al máximo la expresión de la humanidad del paciente; de ahí que sea licito, en ocasiones, suspender intervenciones clínicas que aunque mejoren el metabolismo del paciente, ofenden su persona. Ese "ofender" es el indicador negativo de que no basta atender desde un solo ángulo y creer que se solucionó el problema. Así, si la aseguradora paga el costo de hospitalización, el asunto no ha quedado concluido ni subsanado y, en los casos más severos, como la muerte por negligencia, no hay recuperación posible; por decirlo de algún modo: se ha perdido un todo del propio universo.

No debe pensarse que esto es mera especulación teórica. Gutiérrez, Mederos, Vásquez y Velázquez (2001, p. 15) encontraron que la despersonalización es evidente cuando se analiza el derecho del paciente a un trato digno, aunque el $69 \%$ de las enfermeras saben que los pacientes tienen derecho a la autonomía y un 55\%, a la intimidad, resulta que más del $70 \%$ considera que no se respeta la decisión e intimidad del paciente, ni se le hacen ofrecimientos diagnósticos y terapéuticos.

Las decisiones complejas -aspecto epistémico del recorte de realidad- que cotidianamente enfrentan los profesionales de enfermería, deben centrarse en la particularidad de los pacientes (la realidad como entidad compleja), los cuidados críticos, la colaboración y el seguimiento de tratamientos médicos que ameritan una elección in situ o, a veces, una acción que produce daño, solo porque que no se puede evitar hacerlo. 
Tabla 1. Programas de Bioética y su concepción de interdisciplina

\begin{tabular}{|c|c|c|}
\hline Tema & Referencia & Citas que aluden a lo holístico \\
\hline $\begin{array}{l}\text { El papel del arte (en este } \\
\text { caso un taller de man- } \\
\text { dalas) en el hecho de fo- } \\
\text { mentar una visión holís- } \\
\text { tica de los médicos. }\end{array}$ & $\begin{array}{l}\text { Potash, Chen y Tsang } \\
\text { (2016). Med Humanit, 42, } \\
\text { 17-25. }\end{array}$ & $\begin{array}{l}\text { A growing trend in medical schools is the introduction of } \\
\text { the medical humanities into a biomedically oriented medical } \\
\text { curriculum. Among its aims, such initiatives seek to nurture } \\
\text { the capacity of doctors to understand the psychosocial im- } \\
\text { pact of disease and to gain increased perspective on pain and } \\
\text { suffering as part of a holistic model of care. The curriculum } \\
\text { also aims to help medical students enhance selfawareness } \\
\text { for both self-care and in order to improve patient care (p. 17). }\end{array}$ \\
\hline $\begin{array}{l}\text { Ejemplo del yoga en pa- } \\
\text { cientes con cáncer para } \\
\text { aliviar y estabilizar pa- } \\
\text { cientes. }\end{array}$ & $\begin{array}{l}\text { Chintamany (2015). Inte- } \\
\text { grative Oncology-Finding } \\
\text { an Indian Solution to Uni- } \\
\text { versal Problems for Holis- } \\
\text { tic Cancer Care!! Indian J } \\
\text { Surg Oncol, 6(4), 323-324. }\end{array}$ & $\begin{array}{l}\text { It is essential to take care of the bio-psycho-social and spir- } \\
\text { itual needs of these patients before, during and after treat- } \\
\text { ment and various complimentary therapies are being } \\
\text { employed to address this issue. This holistic approach is re- } \\
\text { quired at all levels of treatment to not only potentiate the } \\
\text { role of established modalities but also to deal with toxicities } \\
\text { related to them (p. 323). }\end{array}$ \\
\hline
\end{tabular}

\begin{tabular}{|c|c|c|}
\hline $\begin{array}{l}\text { Método de bioética que } \\
\text { busca evitar los "ismos". }\end{array}$ & $\begin{array}{l}\text { Schmidt (2008). Método de } \\
\text { interpretación y análisis } \\
\text { holístico en casos bioéticos. } \\
\text { Acta Bioethica, 14(1). }\end{array}$ & $\begin{array}{l}\text { Se propone un "método" fenomenológico y una comprensión } \\
\text { hermenéutica que parte de un paradigma antropoético, de- } \\
\text { liberativo y crítico, el cual permite sistematizar e interpre- } \\
\text { tar las diversas situaciones desde una ética de mínimos a una } \\
\text { de máximos. } \\
\text { Esta propuesta se implementa a través de un método científi- } \\
\text { co que consta de tres estadios: (a) ver-planteamiento, (b) juz- } \\
\text { gar-valoración desde diversos puntos de vista (social, técnica, } \\
\text { jurídica y bioética) e interpretación holística, y (c) actuar-las } \\
\text { respectivas conclusiones e implicaciones del dilema (p. 40). }\end{array}$ \\
\hline $\begin{array}{l}\text { Trabajo en cáncer inter- } \\
\text { disciplinario. }\end{array}$ & $\begin{array}{l}\text { Cadet, Davis, Elks y Wilson } \\
\text { (2016). A Holistic Model of } \\
\text { Care to Support Those Liv- } \\
\text { ing with and beyond. Can- } \\
\text { cerHealthcare, 4, p. } 88 .\end{array}$ & $\begin{array}{l}\text { Providing holistic care is a component of providing integra- } \\
\text { tive care. Integrative care utilizes conventional and comple- } \\
\text { mentary approaches to cancer integrating them with a focus } \\
\text { on the whole person, which includes emotional, spiritual, so- } \\
\text { cial, and lifestyle (diet, physical activity, sleep, relationships) } \\
\text { factors, the provider-patient relationship as a partnership } \\
\text { and interprofessional collaboration (p. 2). }\end{array}$ \\
\hline
\end{tabular}

Fuente: elaboración propi).

Por ejemplo, Sile, Tang, Wadensten y Ahlstrom (2008) reportan que las enfermeras suecas sufren del síndrome de burn out, que está relacionado con los dilemas éticos que enfrentan en la práctica (perplejidad moral) y refieren el testimonio de una enfermera: "Well, the classic case, I must say, is when a patient comes in and is in a bad way and can't eat. Do you give no treatment, or do you put the patient on a drip and use a probe and all that? I think that's dreadfully difficult (nurse 8)".7

7 "Bueno, el caso clásico, debo decir, es cuando un paciente llega en mal estado que le impide comer. No se le da tratamiento o solo instalas una venoclisis y administras solución gota a gota al paciente y, ¿eso es todo? Pienso que eso es muy difícil". 
Sin expresarlo en términos técnicos, la enfermera habla de la dificultad de aplicar el principio de sindéresis, "haz el bien y evita el mal" en la realidad total del paciente, que no es la suma de las intervenciones posibles. A veces no podemos hacer el bien deseable o evitar los males (Gómez, 2010).

\section{Conclusión}

La enfermería es un excelente ejemplo de una actividad humana que refleja una realidad compleja, lo que epistémicamente también es complejo. Al revisar los artículos sobre el concepto de ciudado y la interdisciplina, en esta última encontramos esa postura, aunque el término complejidad no se utilice como tal.

La enfermería descubre que la noción de "cuidado" implica un aspecto antropológico: la dignidad humana es un todo complejo, no se le puede reducir ni explicar solo por la "autonomía", las relaciones sociales o cualquier categoría aislada. Los artículos revisados muestran que se completa o atiende mejor el bien humano cuando se reconoce que, ese bien buscado, no es la suma de partes, sino un todo organizado que se atiende al mismo tiempo.

Asimismo, hay necesidad de hacer explícitos los fundamentos ontológicos del cuidado. Parece útil la concepción tradicional de la analogía para hacer explicitas las distintas características del cuidado. También es importante reconocer que las nociones antropológicas de lo que es el hombre son indispensables para entender el papel de los enfermeros en la actividad profesional.

El cuidado es una categoría mental que cuando se busca encontrar su lugar, se descubre que no está en ninguno de ellos. Es de un modo semejante a la categoría del pensar.
Es tan general dentro de las categorías de la razón práctica moral que está casi al mismo nivel de la sindéresis.

Al revisar la literatura reciente, se descubre una exigencia en los cuidados de tipo holístico o sea de un modo complejo. Los autores, no siempre utilizan esa palabra, pero mantienen su sentido.

La enfermería comparte con la bioética una peculiaridad: la interdisciplinariedad; requiere, además, habilidades de ver un problema en visión simultánea con otras disciplinas, para así tomar una decisión adecuada. La bioética análogamente necesita el consejo de muchas áreas; esa peculiaridad da la impresión de no ubicarla en ningún lado.

La perspectiva ética es el ejemplo que mejor muestra ese cuidado de la enfermería. En el fondo de los ejemplos citados subyace la irremediable consideración ética, como el gran concepto que guía el cuidado; es decir, el cuidado no es "neutro", siempre tiene en consideración un marco antropológico que subyace y dirige la mirada a ese todo que es el ser humano y que, al cuidarlo, se requiere mantener el todo y a la vez entender sus partes dinámicas, para que dentro de lo inestable y cambiante que es el cuidado, procurar el mayor bien a esa persona concreta en ese tiempo específico. Todo esto con la idea de visualizar y tener en cuenta esos elementos, que es lo que distingue a la enfermería como profesión. Así pues, hay una consciencia sobre las técnicas y, sobre todo, de la visión del todo que la convierte en profesión, pero que siempre es interdisciplinaria.

\section{Referencias}

Álvarez Suárez, J. L., Del castillo Arévalo, F., Fernández Fodalgo, D. y Muñoz Melén- 
dez, M. (2010). Manual de valoración de patrones funcionales. Gijón: Dirección de enfermería de atención primaria.

Aristóteles. (1987). Metafísica. Madrid: Gredos.

Bernal-Orozco, M. F., Bizmanos, B. y Celis de la Rosa, A. J. (2008). La nutrición del anciano como un problema de salud pública. Antropo, 16, 43-55.

Cadet, T., Davis, C., Elks, J. y Wilson, P. (2016). A Holistic Model of Care to Support Those Living with and beyond. Healthcare. 4(4), 88.

Calderón, D. (2003). Epistemología de la vida. Medicina y Ética, XIV(2), 191-201.

Careplans.com (2017). Care Plans. Recuperado de https://www.careplans.com/blog/ index.php/acute-pain-care-plan/

Chintamany (2015). Integrative Oncology-Finding an Indian Solution to Universal Problems for Holistic Cancer Care!! Indian J Surg Oncol, 6(4), 323-324.

Delgado, M., Hernández, C. y Ostiguín, R. (2007). Fundamentos filosóficos de la propuesta de Virginia Avenel Henderson. Enfermería Universitaria, 4(1). Recuperado de http://www.revistas.unam. mx/index.php/reu/article/view/30303

Furstenberg, C. (2015). La empatía a la luz de la fenomenología: perspectivas en el contexto del cuidado. Revista Latinoamericana de Bioética, 2(29), 26-41.

Feyerabend, P. (1993). Expertos en una sociedad libre. ¿Por qué no Platón? Madrid: Tecnos.

García, R. (2009). Sistemas complejos. Barcelona: Gedisa.
Gómez, J. E. (2010). La autonomía del menor, bioética y religión: fuente de paradojas. Boletín Médico del Hospital Infantil de México, 67(3), 223-229.

Gómez, J. E. (2014). El consentimiento informado en los procedimientos de laboratorio: calidad y respeto en primer nivel de atención. En O. Márquez Mendoza, M. Veytia López y R. Guadarrama Guadarrama (Comps.). Reflexiones latinoamericanas en Bioética (pp. 143-156). México, D. F.: Universidad Autónoma del Estado de México [UAEM], Torres Asociados.

González, A. M. (2001). Claves éticas para la Bioética. Cuadernos de Bioética, XII(46), 305-320.

González, Á. (1987). Tratado de Metafísica. Madrid: Gredos.

Gutiérrez, R. L., Mederos, S. E., Vásquez, O. M. y Velázquez, M. A. (2001). Derechos del paciente hospitalizado: Responsabilidad en la práctica de enfermería. Revista de Enfermería del Instituto Mexicano del Seguro Social, 9(1), 15-18.

Jawad F. y Kalra S (2016). Ramadan and diabetes: Holistic trial design. Journal of $\mathrm{Pa}$ kistan Medical Association, 66(7), 791-2. Recuperado de https://www.ncbi.nlm. nih.gov/pubmed/27427122

Maliandi R., Thüer O. y Ceccheto, S. (2009). Los paradigmas de fundamentación en la ética contemporánea. Acta Bioethica, 15(1), 11-20.Recuperado de www.scielo. cl/pdf/abioeth/v15n1/art02.pdf

McIntyre, A. (2001). Animales racionales y dependientes. Barcelona: Paidós.

Mitchel, M. (2009). Complexity a guided tour. Nueva York: Oxford University Press. 
Pessina, A. (2007). La cuestión del método desde la perspectiva de la bioética personalista. En M. Tarasco (Ed.), Comités hospitalarios de bioética (pp. 67-74). México, D. F.: Manual Moderno.

Potash, J. S., Chen, J. Y. y Tsang, J. P. Y. (2016). Medical student mandala making for holistic well-being. Medical Humanities, 42, 17-25.

Redmond, W. (1999). Lógica simbólica para todos. México, D. F.: Universidad Veracruzana.

Reyes, E. (2009). Fundamentos de enfermería. México, D. F.: Manual Moderno.

Schmidt, L. (2008). Método de interpretación y análisis holístico en casos bioéticos. Acta Bioethica, 14(1), 39-46.

Sile, M., Tang, P. F., Wadensten, B. y Ahlstrom, G. (2008). Workplace distress and ethical dilemmas in neuroscience nursing. The Free Library. Recuperado de https://www.thefreelibrary.com/Work- placedistressandethicaldilemmasinneurosciencenursing.-a0183489363

Sokal y Bricmont (1999). Imposturas intelectuales. Barcelona: Paidós.

U. S. National Library of Medicine (2017). Pubmed. USA. Recuperado de https:// www.ncbi.nlm.nih.gov/pubmed?ter$\mathrm{m}=$ holistic\%5BTitle\%5D

Washington et al. (2017). A multimethod analysis of shared decision-making in hospice interdisciplinary team meetings including family caregivers. Palliat Med, 30(3), 270-278.

Younas, A. y Sommer, J. (2015). Integrating nursing theory and process into practice; Virginia's Henderson Need Theory. International Journal of Caring Sciences, 8(2), 443-450.

Zamanzadeh, M., Valizadeh, L. y Keogh, B. (2015). Effective Factors in Providing Holistic Care: A Qualitative Study Indian. Journal of Palliative Care, 21(2), 214-224. 